\title{
Pulmonary Arterial Hypertension Associated Systemic Sclerosis
}

\author{
Attapon $\mathrm{C}^{1,2 *}$ and Ruangrong $\mathrm{C}^{3}$ \\ $110^{\text {th }}$ Zonal Tuberculosis and Chest Disease Center, Chiang Mai, Thailand \\ 2Department of Disease Control, Ministry of Public Health, Thailand \\ ${ }^{3}$ Department of Pathology, Faculty of Medicine, Chiang Mai University, Chiang Mai, \\ Thailand
}

Editorial

Volume 1 Issue 2

Received Date: February 27, 2017

Published Date: April 03, 2017

*Corresponding author: Attapon Cheepsattayakorn, Department of Disease Control, Ministry of Public Health, Thailand, Tel: 6653 140767, 6653 276364; Fax: 6653 140773, 6653 273590; Email: Attapon1958@gmail.com; attaponche@yahoo.com

\section{Editorial}

Pulmonary hypertension (PAH) affects up to $12 \%$ of patients with systemic sclerosis (SSc), with mortality rate of $50 \%$ within 3 years. Patients with SSc-associated PAH (SSc-PAH) have three times increased risk of death, compared to idiopathic PAH (IPAH). SSC-PAH patients may receive a diagnosis late in the course of disease due to insidious onset and the high prevalence of musculoskeletal, cardiac, and pulmonary parenchymal comorbidities. Conventional therapies often yield poor results, compared to IPAH cohorts. The exact reasons behind this include variations in the pathologic mechanisms, inadequate strategies to detect and treat SSc-PAH early in its course, and differences in cardiovascular response to increasing afterload. The optimal screening methods are yet unknown, but the 6minute walk test, transthoracic echocardiography, and magnetic resonance imaging, each has distinctive advantages and disadvantages. The Dopplerechocardiography optimal thresholds are still subject to investigation, particularly in patients with mild PAH. Right cardiac catheterization remains the confirmatory method for all suspected SSc-PAH cases. Further investigations are urgently needed for identification of the optimal screening methods with highest sensitivity and specificity. 